(2) $\begin{array}{r}2017 \text { Volume 23(5): 687-708 } \\ \text { doi:10.3846/20294913.2015.1077902 }\end{array}$

\title{
CAN RESIDENTIAL INTEGRATION BE A STABLE EQUILIBRIUM?
}

\author{
Raúl CONEJEROS ${ }^{\mathrm{a}}$, Miguel VARGAS ${ }^{\mathrm{b}}$ \\ ${ }^{a}$ Escuela de Ingeniería Bioquímica, Pontificia Universidad Católica de Valparaíso, Valparaiso, Chile \\ ${ }^{b}$ Departamento de Economía, Universidad Diego Portales, Avenida Santa Clara 797, Santiago, Chile
}

Received 21 March 2012; accepted 29 June 2014

\begin{abstract}
Shelling's model and its different versions predict that segregation is the unique stable equilibrium. However, cities around the world show different levels of intermingle, and it is very uncommon to observe fully segregated urban patterns even when segregation is high. We argue that individuals do not take into account only their neighbours characteristics for making a location decision. In particular, if residential segregation generates ghettos of low-skilled labourers, the societal level of production would diminish and, consequently, individuals' level of consumption too, affecting all society members' well-being. When agents care about the impact of segregation on their own consumptions, we prove that the set of system's equilibria can be one containing states of residential integration. Besides, using simulations, we compare the aggregated utility related to these equilibria with the maximum level of aggregated utility.
\end{abstract}

Keywords: segregation, agent-based, artificial economics.

JEL Classification: R0, R2, C15.

\section{Introduction}

Studies by Schelling (1971) of segregation based on prejudice show that segregation arises spontaneously and that it is the only stable equilibrium that society can reach, even if each member of this society may prefer living in a mixed community.

After Schelling's work, several new investigations have confirmed that result. For instance, Clark (1991) and Ruoff (2006) empirically tested Schelling model predictions and they concluded that stable integrated equilibria are very unlikely.

Similarly, using regression discontinuity methods and data from Sweden, Alden et al. (2014) empirically showed that, after non-European immigrants reach a tipping point in a neighbourhood native population, growth discontinuously drops, a behaviour that is likely to be related to ethnic and socio-economic segregation.

Corresponding author Miguel Vargas

E-mail: miguel.vargas@udp.cl 
Zhang (2004) is an interesting work because it provides the first successful formal analytical framework for Schelling-like models. He uses stochastic evolutionary game theory to prove that segregation is the unique stable equilibrium. He assumes that individuals have preferences for living in integrated communities, and even under these setting segregation arises as equilibrium. Zhang and Zheng (2015) provide empirical evidence that even in a segregated country as the United States of America (U.S.A.), people are willing to pay for reducing segregation.

Pancs and Vriend (2007) reinforces Zhang (2004) result, showing that this is true even when all agents have a strict preference for living in a fully integrated society. Even more, they indicate that this result raises doubts about the ability of public policies to produce urban integration. The key elements behind these results are the presence of externality and the asymmetry of the utility function: as long utility functions favour majority over minority, segregation will arise.

Zhang (2011) revisits his previous investigations and concludes that segregation arises almost all the time in a checkerboard model because tipping is less probable to occur to such residential patterns.

Although these are very strong results we argue that they do not represent all possible segregation patterns that can be empirically observed. For instance, if we consider the U.S.A. metropolitan areas, there are examples of moderate segregation. Accordingly to The Social Science Analysis Network calculations, based on census data, the average Dissimilarity index for white and black individuals in the U.S.A. is 58.5 (the maximum value is 100). Furthermore, $25 \%$ of the American metropolitan areas have a Dissimilarity index, which is less than 50, and most of them have a Dissimilarity index of 60, as it can be appreciated in Figure 1.

Bayer et al. (2004), using micro-data from the San Francisco bay area, found evidence that both level and drivers of racial segregation in USA depend on the kind of race. For instance when Hispanic population reaches higher levels of income the propensity for living amongst Hispanics is reduced, something that does not happen in the Afro-American case, where segregation is higher and it persists even when Afro-Americans reach higher

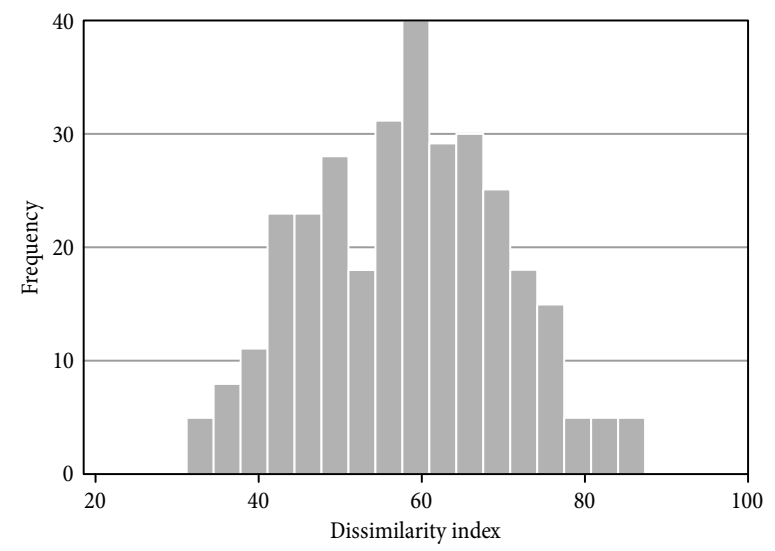

Fig. 1. American metropolitan areas dissimilarity index histogram 
levels of income. This result is ratified by Bayer et al. (2014). Hence, in a dynamic setting segregation based on race varies and it can be reduced for some ethnic groups when some of their socio-economics characteristics change.

Given the potentially important policy consequences that these results would have, it is relevant to find an answer for these differences between theoretical prediction and empirical observations.

Grauwin et al. (2009) developed a formal Schelling-like model, which includes the possibility of altruism as a parameter weighting the individual utility and the collective utility. The closer to 1 the parameter is, the more altruistic agents are. This altruism is understood as controlled by a central planner via a sort of pigouvian tax. When this parameter is close to the one they find equilibria associated with low levels of segregation. Grauwin et al. (2012) expands the analysis to different kind of utility functions and they study the equilibrium characteristics of them. These two works have added significant insights to the understanding of Schelling-like models and social interactions. Besides, they have given new and solid theoretical background for the analysis of segregation's public policies.

Although Grauwin et al. (2009) and (2012) found situations where integration is an stable equilibrium they are based on the introduction of a pigouvian tax. However, empirically it is possible to observe moderate and low segregation levels without this tax.

Other interesting works reporting equilibria different from segregation are Bischi and Merlone (2011) and Singh et al. (2009). The former develop a discrete two-dimensional dynamic model and then numerically analyse the system's behaviour. They found path-dependence, i.e., the long-run collective behaviour depends on the initials conditions and the historic accidents, which would have through the trajectories. According to them “... integration is possible if the system starts with a sufficiently balanced initial mixture of the two populations." Singh et al. (2009) presents results for different artificial cities' size and agents' tolerance and density parameters. The most striking finding of this investigation is that considering the same values as Schelling's, segregation is strictly a small city phenomenon, which means that as long as the number of agents increases, the conditions needed for observing segregation as an equilibrium become more demanding. Specifically, in order to obtain a segregated city the disparate comfort threshold must be greater than 3 when the city is greater than $8 \times 8$, the values originally used by Schelling.

Xie and Zhou (2014) introduces individual-level agents heterogeneity in racial tolerance. Their main finding is that in the long run the model reaches a low level of segregation equilibrium than would be observed with homogenous racial tolerance.

There are two main differences between the works commented above and the investigation presented here: first, instead of analysing the system behaviour and its computational characteristics, our focus is on agents' economic motivations and how they can lead to a particular segregation pattern, and, second, we analytically investigate about the system's equilibrium instead of doing it numerically.

Our hypothesis is that individuals do not take into account their neighbours features only when they make their location decisions, but they can consider the potentially negative effects that segregation can have on their own level of consumption. Regarding negative effects literature has pointed out that segregation can have a negative impact on the 
segregated population through the level of unemployment, out-of-wedlock births, criminality, low educational achievement, income inequality, and poverty traps, among others. Hence, if one part of society is affected by this sort of difficulty, it is almost certain that the population as a whole will be affected as well. For example, if segregation has a negative impact on educational achievement, ghettos of low-skilled labourers will emerge, a process, which can reinforce itself. Consequently, society will lose productivity and, therefore, the level of consumption of all individuals will decrease, diminishing the welfare of every single individual, regardless of whether they are segregated or not. If that is the case, the prejudiced population is going to face a trade-off between the desire for living just among peers and the lower level of consumption that segregation brings about. It would be also the case that segregation generates higher levels of violent crime $^{1}$, which will affect society as whole. Traditional Schelling-like models do not include these aspects. Probably the unique work that incorporates elements of this sort is Conejeros and Vargas (2007). In this article an agent-based model is developed in order to simulate a Schelling-like model including very simple production and consumption functions. The main finding they obtain shows that integration can be a stable equilibrium. Conejeros and Vargas (2007) argues that the negative consequences of segregation would also affect the utility of the non-segregated population. The idea behind this statement is that the negative effects of segregation on the segregated population in the long run will become a cost for society as whole, and if all members of society are aware of these costs, integration may arise as a stable equilibrium.

Although Conejeros and Vargas (2007) results are interesting, more general conclusions cannot be made as it pertains to only one particular set of parameter values. Therefore, based on this argument we develop a formal model to analytically show which conditions determine social integration to be a stable equilibrium.

This work has followed the traditional approach, which says that segregation can have negative effects on individuals, although we are aware of those works that using the Moving to Opportunity Program data have conclude that segregation has negligible effects upon households, such as, for instance, Kling and Liebman (2004), Kling et al. $(2005,2007)$ and Ludwig et al. $(2008,2013)$ and others that question the negative effects of segregation, such as Bolt et al. (2010) whom argue that there is not a strong relationship between segregation and integration. However we believe that this is still a matter for discussion, as shown by Clampet-Lundquist and Massey (2008) and Ludwig et al. (2008), and others works such as Ananat (2011) and Corvalan and Vargas (2015), whom have found negative effects of segregation on income distribution and the level of conflict observed in a country.

In Section 1, the theoretical model is developed, explaining its main characteristics. Then, in Section 2 the main results of the model are presented. Section 3, through the use of simulations, we exemplify equilibria and compare the relationship between equilibria and the maximum level of aggregated utility. Finally, conclusions and final remarks are offered.

\footnotetext{
${ }^{1}$ Bjerk (2010) develops a model and then tests empirically the hypothesis that segregation increases the level of violent crime finding evidence that support this hypothesis.
} 


\section{The model}

The model developed here is based upon Zhang (2004) and Conejeros and Vargas (2007). As in Conejeros and Vargas (2007), we add to Zhang's model three new features: first we introduce a consumption term into the agents' utility function, hence in our version of the model agents have a two-terms utility function, one of them related to location, as in Zhang (2004), and another one related to consumption. The second feature is that agents may be of two different types: disadvantaged and non-disadvantaged. Segregation literature has been focused mainly on studying this phenomenon related to income and race, both highly correlated. Consequently, we have considered two types of population, as it was mentioned above, one non-disadvantaged and another one disadvantaged. The non-disadvantaged one may represent a dominant group within society such as affluent population or racial majority, like white Anglo-Saxon in U.S.A. meanwhile the disadvantaged group may represent a racial minority, like Afro-American people in U.S.A or low-income households. The third feature is that agents have a very simple production function. This production function will depend on two factors, first, if the agent is a non-disadvantaged or disadvantaged one and, second, on the quantity of agents of the same type that live in the same neighbour.

The first step is to define an artificial society comprising an advantaged prejudiced population and a non-prejudiced disadvantaged population. Each agent belongs to just one of these two population groups. The proportion of these two populations is given by $\pi_{j}$, with $j \in\{0,1\}$ indexing the population's types. If $j=1$, the agent is a disadvantaged one and with $j=0$ a non-disadvantaged one. Each one of this society's members, or agents, is allocated in the vertex of an $N \times N$ lattice graph with a periodic boundary condition.

Utility. Each agent's utility is made up of two parts: a deterministic one $u_{i}$ and a stochastic term $\epsilon$. The deterministic part depends on how many like-type neighbours he has in the local neighbourhood - a neighbourhood here is a Moore neighbourhood: a simple $3 \times 3$ square with output cell in the middle - and on his level of consumption. The stochastic part reflects other relevant neighbourhood characteristics and is assumed to be independent of the agent and location. As Zhang (2004) points out, the latter is because agents value different characteristics, and different locations have different idiosyncratic traits. Following McFadden (1973), the random component corresponds to unobserved utility terms, and if it is assumed to be independently distributed and with a cumulative extreme value distribution, the swap probability among agents will reduce to the analytically convenient logit form, as it will be shown later on.

The deterministic utility function $u$ is assumed additively separable in two components: a location term $\ell$ and a consumption term $C$. Location term is based on Zhang (2004) and corresponds to the following expression:

$$
\ell=\left\{\begin{array}{lc}
Z\left(\frac{x}{n}\right), & \text { if } x \leq n \\
(2 Z-M)+(M-Z)\left(\frac{x}{n}\right), & \text { otherwise }
\end{array},\right.
$$

where $Z>M>0$ are parameters guaranteeing a linear kinked shape, increasing on the left 
side of $n$ and decreasing on the right, with the peak at $n$, as shown in Figure 2. Therefore, $n$ is the number of like-type neighbours in the local neighbourhood which maximizes $\ell$. The total number of like-type neighbours of the local neighbourhood is $2 n$. $x$ is the actual number of like-type neighbours in the local neighbourhood.

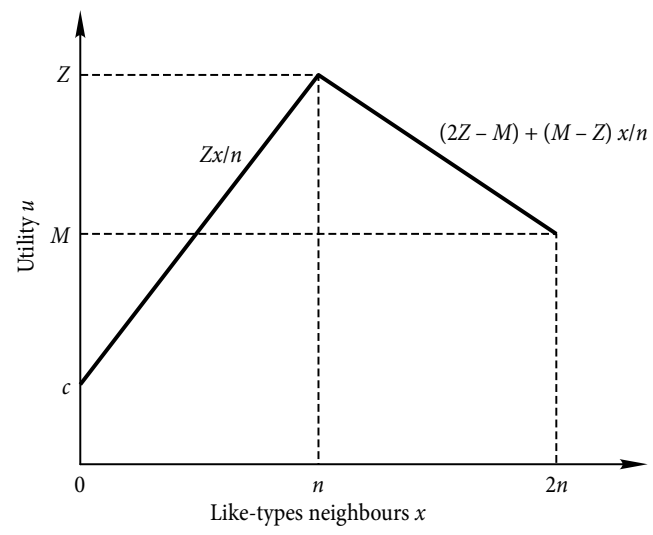

Fig. 2. Deterministic utility

The consumption term for both types of agent is just the normalised level of consumption. Therefore, the deterministic term of the utility function is given by:

$$
u=\ell+\alpha C,
$$

with $\alpha$ being a positive parameter indicating the importance of consumption for agents. The utility function for any agent $i$ of type $j$ is:

$$
U_{i j}=\beta u_{j}+\epsilon .
$$

Where $\beta$ indicates how important is the deterministic part of the utility function related to the stochastic one. Finally, the aggregate utility is:

$$
U=\sum_{1}^{N^{2}} U_{i} .
$$

Production. Each agent is endowed in every period of time with 1 unit of "productivity" that is not cumulative. An important assumption made here is that the agent's productivity can be affected by the local neighbourhood's characteristics (group effects). In particular, it is assumed that if in a local neighbourhood the number of disadvantaged agents is greater than a threshold value $\tau$, then the productivity of that type of agent decreases. This argument follows Benabou (1996), which says that when low-income group is segregated their local public goods consumption will diminish, because, for instance, they are funded by local taxes. Whether education is locally funded, then low-income municipalities will provide low quality education, which in turn will affect the qualifications and productivity of segregated low income households. Peer effects may reinforce this process. Hence, we have considered the productivity of a disadvantaged agent as follow: 


$$
p_{1}=\left\{\begin{array}{ll}
\frac{x-2 n}{\tau-2 n}, & \text { if } x>\tau \\
1, & \text { otherwise }
\end{array},\right.
$$

where $n \leq \tau<2 n$ is the threshold value of like-type neighbours for triggering the productivity diminishing process, as is depicted in Figure 3.

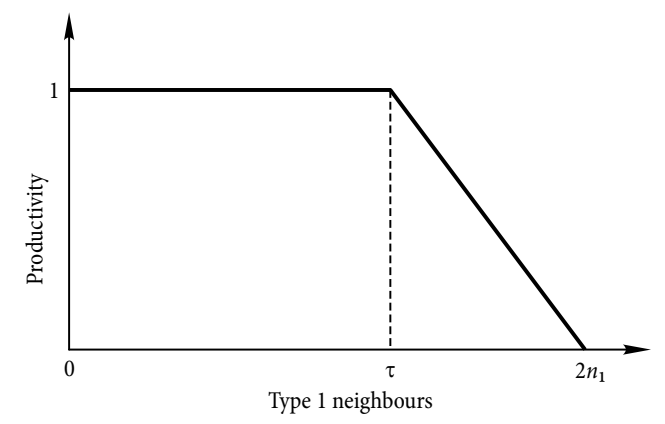

Fig. 3. Productivity function of disadvantaged agents

The productivity of a non-disadvantaged agent is always 1 . The aggregate production is:

$$
P=\sum_{1}^{N^{2}} p_{i}
$$

The level of consumption of every agent is $C=\mathrm{P} / N^{2}$. This means that all the agents, prejudiced and non-prejudiced, are going to be affected negatively by segregation. We are aware this is a strong assumption and a simplification, but it can be considered as the consumption of a public good provided by the state, of which the provision will depend on the level of production of the economy, because, for example, it is funded by taxes. Hence, the lower the production of the economy, the lower the consumption will be.

Log-linear behavioural rule. A log-linear behaviour is observed when agents change their location based on their own personal interests. In each period, two agents coming from different neighbourhoods are selected and in order to perform a change, every agent will bid in a sort of auction for a better location; consequently the focus is on the sum of two chosen agents' utility. If the sum of switching is bigger than the sum of not switching, then agents will swap their locations. If the switch situation is called $S$ and the opposite NS, and, for the following exposition, the chosen agents are agent 1 and agent 2 , and they do not swap locations, then:

$$
U_{1}(\cdot \mid N S)+\epsilon_{1}+U_{2}(\cdot \mid N S)+\epsilon_{2}=U_{1}(\cdot \mid N S)+U_{2}(\cdot \mid N S)+\epsilon_{1}+\epsilon_{2}=V^{N S}+\eta,
$$

but if they do, then:

$$
U_{1}(\cdot \mid S)+\epsilon_{1}+U_{2}(\cdot \mid S)+\epsilon_{2}=U_{1}(\cdot \mid S)+U_{2}(\cdot \mid S)+\epsilon_{1}+\epsilon_{2}=V^{S}+\varepsilon .
$$


Agents will change location if and only if $V^{N S}+\eta<V^{S}+\varepsilon$. It is assumed that $\eta$ and $\varepsilon$ are independent, and that they follow an identical extreme value distribution. Then, based on McFadden (1973), and following Zhang (2004), a log-linear switch rule can be established:

$$
\operatorname{Pr}(S)=\left(\frac{e^{V^{N S}}+e^{V^{S}}}{e^{V^{N S}}}\right)
$$

This behavioural rule depends only on the deterministic utilities. Therefore, it is possible to avoid taking into account the stochastic utilities, which are unobservable.

A potential function. Let us consider the set $E D$ as the set of all edges that connect two agents of different type. A function $\rho$ is defined as the cardinality of set $E D$. This function, once it has been normalised, can be used as a segregation measure.

Lemma 1. Let us consider the function $\Phi$ as

$$
\Phi(\cdot)=\left\{\begin{array}{cl}
\rho+\alpha C, & \text { if } x_{1} \geq \tau \text { and } \alpha \text { is large enough } \\
-\rho, & \text { otherwise }
\end{array},\right.
$$

then, $\Phi$ is a potential function for this spatial game.

Proof. Monderer and Shapley (1996) define a general ordinal function $\Phi$ for a game in strategic form $\Gamma\left(u^{1}, u^{2}, \ldots, u^{n}\right)$ with finite number of players $n$, a set of strategies for the player $i, Y^{i}$, and a payoff function $u^{i}$, such as if for every player $i$ and for every $y^{-i} \in Y^{-i}$ we have that $u^{i}\left(y^{-i}, x\right)-u^{i}\left(y^{-i}, z\right)>0 \Leftrightarrow \Phi\left(y^{-i}, x\right)-\Phi\left(y^{-i}, z\right)>0$ for every $x, z \in Y^{i}$.

Consequently in order to prove Lemma 1 we need to show that $\Delta u>0 \Leftrightarrow \Delta \Phi>0$.

When 2 like-type agents interchange their locations, the residential pattern does not change. Hence, the production of society, consumption and $\rho$ do not change. Therefore we will put our attention on those cases where agents of different type interchange their locations. The effects of these changes will depend on $x_{1}$ and $\alpha$. We have 4 cases: $x_{1} \geq \tau$ and $\alpha$ large, $x_{1} \geq \tau$ and $\alpha$ small, $x_{1}<\tau$ and $\alpha$ large, and $x_{1}<\tau$ and $\alpha$ small. Where we understand that $\alpha$ is large when the consumption part of the utility function is more important than the location one. When $x_{1}<\tau$ regardless of $\alpha$ the production function is not affected, therefore the level of consumption is not affected either. As consumption is not affected, when we calculate $\Delta \Phi$ the consumption components cancel each other, then the relevant potential function is $-\rho$. This case is as the one studied by Zhang (2004) whom proves that under these conditions $-\rho$ fulfils all the potential function requirements.

When $x_{1} \geq \tau$ and $\alpha$ is large enough we have two main types of changes: the first one is when the disadvantaged agent is minority in the neighbourhood and after the interchange becomes part of the majority, and the second one is when the disadvantaged agent is already part of the majority and after the interchange the disadvantaged agents' share in the neighbourhood increases. Before describing these interchanges note that when $x_{1} \geq \tau$ the type 1 agent production decreases and hence the level of consumption for one agent is $(x-2 n) /\left(N^{2}(\tau-2 n)\right)$.

Interchange of Type 1: from minority to majority. Figure 4 depicts the case where a disadvantaged agent belonging to the minority in a neighbourhood, after an interchange becomes 


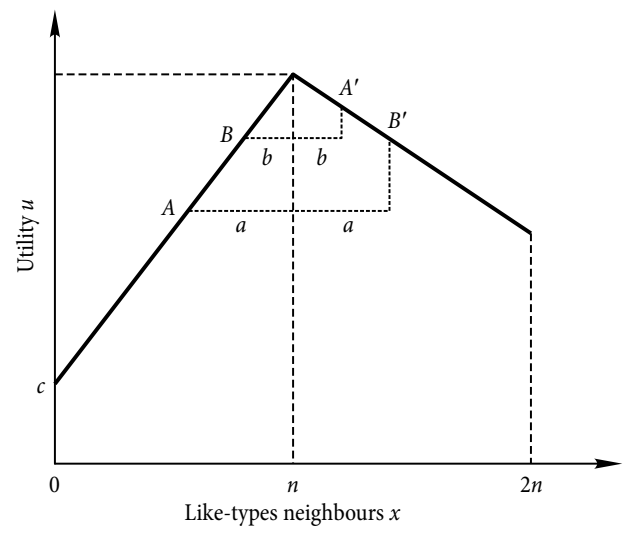

Fig. 4. Interchange of Type 1

part of the majority. As a matter of fact in this case both agents are in the same situation. Table 1 shows the utility values when a disadvantaged agent becomes part of the majority in the neighbourhood. The two first columns contain the utility values when the disadvantaged agent starts at point $A$ (see Fig. 4), and the two finals columns when she/he starts at point $B$ (see Fig. 4). When type 1 agent starts at point $A$ the utility variation for her/him is:

$$
(2 Z-M)+(M-Z) \frac{(n+b)}{n}-Z \frac{n-a}{n}+\alpha\left[\frac{(n+b)-2 n}{N^{2}(\tau-2 n)}-C\right] .
$$

Table 1 . Type 1 interchange utility variation

\begin{tabular}{lcc}
\hline $\begin{array}{l}\text { Loca- } \\
\text { tion }\end{array}$ & Utility (agent of type 1 starts in $A)$ & Utility (agent of type 1 starts in $B)$ \\
\hline$A$ & $\frac{Z(n-a)}{n}+\alpha C$ & $\frac{Z(n-a)}{n}+\alpha C$ \\
\hline$B$ & $\frac{Z(n-b)}{n}+\alpha C$ & $\frac{Z(n-b)}{n}+\alpha C$ \\
\hline$A^{\prime}$ & $(2 Z-M)+\frac{(M-Z)(n+b)}{n}+\alpha\left[\frac{(n+b)-2 n}{N^{2}(\tau-2 n)}\right]$ & $(2 Z-M)+\frac{(M-Z)(n+b)}{n}+\alpha\left[\frac{(n+a)-2 n}{N^{2}(\tau-2 n)}\right]$ \\
\hline$B^{\prime}$ & $(2 Z-M)+\frac{(M-Z)(n+a)}{n}+\alpha\left[\frac{(n+b)-2 n}{N^{2}(\tau-2 n)}\right]$ & $(2 Z-M)+\frac{(M-Z)(n+a)}{n}+\alpha\left[\frac{(n+a)-2 n}{N^{2}(\tau-2 n)}\right]$ \\
\hline
\end{tabular}

For the agent of type 0 the utility change is:

$$
(2 Z-M)+(M-Z) \frac{(n+a)}{n}-Z \frac{(n-b)}{n}+\alpha\left[\frac{(n+b)-2 n}{N^{2}(\tau-2 n)}-C\right] .
$$

Summing up both agents' utility changes we get:

$$
\frac{M}{2 n} 2(a+b)+2 \alpha\left[\frac{(n+b)-2 n}{N^{2}(\tau-2 n)}-C\right] \text {. }
$$


Proceeding in the same fashion for the case where the agent of type 1 starts in $B$ we get:

$$
\frac{M}{2 n} 2(a+b)+2 \alpha\left[\frac{(n+a)-2 n}{N^{2}(\tau-2 n)}-C\right] .
$$

The first part of expressions 10 and $11,2(a+b)$, corresponds to the decrease in the total number of different type neighbours multiplied by the constant $M / 2 n$. The second part is the decrease in the consumption multiplied by $\alpha$.

Interchange of Type 2: A larger agent of type 1 share. Figure 5 depicts the case when the agent of type 1 is already part of the majority in the neighbourhood and after the swaps her/his share increases, i.e. she/he starts in $D$ and ends up in $D^{\prime}$. Table 2 shows the agents' utility values in this case. Agent of type 0 utility variation is:

$$
Z \frac{(c-d)}{n}+\alpha \frac{(d+c)}{N^{2}(\tau-2 n)}
$$

Table 2. Type 2 interchange utility variation

\begin{tabular}{cc}
\hline Location & Utility (agent of type 1 starts in D) \\
\hline$C$ & $\frac{Z(n-c)}{n}+\alpha\left[\frac{(n+d)-2 n}{N^{2}(\tau-2 n)}\right]$ \\
\hline$C^{\prime}$ & $\frac{Z(n-d)}{n}+\alpha\left[\frac{(n+c)-2 n}{N^{2}(\tau-2 n)}\right]$ \\
\hline$D$ & $(2 Z-M)+\frac{(M-Z)(n+d)}{n}+\alpha\left[\frac{(n+d)-2 n}{N^{2}(\tau-2 n)}\right]$ \\
\hline$D^{\prime}$ & $(2 Z-M)+\frac{(M-Z)(n+c)}{n}+\alpha\left[\frac{(n+c)-2 n}{N^{2}(\tau-2 n)}\right]$ \\
\hline
\end{tabular}

and the utility change for agent of type 1 is:

$$
(M-Z) \frac{(c-d)}{n}+\alpha \frac{(d-c)}{N^{2}(\tau-2 n)} .
$$

Summing up these two utility changes we get:

$$
\frac{M}{2 n} 2(c-d)+\alpha \frac{2 d}{N^{2}(\tau-2 n)} .
$$

First part of expression 14 is the decrease of the total number of different types neighbours and the second part is the decrease in the consumption due to the change in the number of same type neighbours.

All the cases analysed and their reverses switches exhaust all different types agents interchanges. As Zhang (2004) does we can inflate $\rho$ by the constant $M / 2 n$ in $\Phi$. Consequently, if an interchange affects the moving agents utility by $\Delta U$, the function $\Phi$ will change in $\Delta U$. 


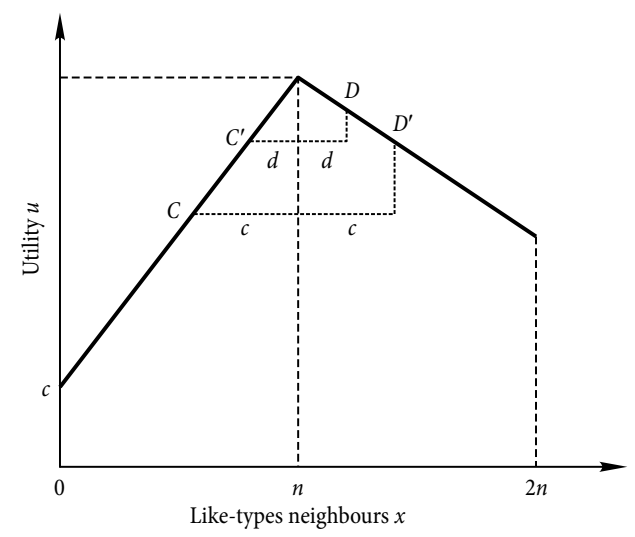

Fig. 5. Interchange of Type 2

When $x_{1} \geq \tau$ and $\alpha$ is small the location part of the utility predominates over the consumption one. In this case the relevant part of $\Phi$ is $-\rho$. It is easy to see that the utility variation will be $\Delta(\rho+\alpha C)$. As $\Delta \alpha C<0$ and $\alpha$ is relatively small, then $\Delta(\rho+\alpha C)>0$ which means that the decrease in the number of different type neighbours will increase the utility. Consequently, $\Delta U>0 \Leftrightarrow \Delta \Phi>0$.

Therefore, $\Phi$ is a potential function of this spatial game with a log-linear behavioural rule.

\section{Results}

We define the function $\sigma: \Lambda_{N} \rightarrow\left\{\right.$ type $_{0}$, type $\left._{1}\right\}$ as a state of the $N \times N$ lattice $\Lambda_{N}$, which identifies each location with its type of occupant. $\sigma^{t}$ represents the state $\sigma$ at the time $t$. Hence, we have a finite Markov process with a transition probability matrix $P^{\beta}$. This markovian process is perturbed due to the shock $\epsilon$ of the utility function. The larger $\beta$ is, the lower the perturbation's effects will be. As there is a positive probability of going from any state to any other state in a finite number of periods, $P^{\beta}$ is irreducible. Besides, as the process can travel from any the state $\sigma$ to $\sigma$ itself in any finite number of periods, $P^{\beta}$ is also aperiodic. Consequently $P^{\beta}$ has a unique stationary distribution $\mu^{\beta}$ satisfying $\mu^{\beta} P^{\beta}=\mu^{\beta}$. The distribution $\mu^{\beta}(\sigma)$ is the cumulative relative frequency with which state $\sigma$ will be observed when the process runs for a long time, and the probability of observing $\sigma$ at any time $t$ when $t$ is large enough.

Let us consider the set $\Sigma$ as the set of all states, and let us define $S$ as a set of states that maximise the potential function $\Phi$, such as $S=\{\sigma \mid \Phi(\sigma) \geq \Phi(y), \forall y \in \Sigma\}$. A finite $N$ guarantees that $S$ is non-empty.

Proposition 1. In this artificial world, $S$ is stochastically stable, i.e., $\lim _{t \rightarrow \infty} \lim _{\beta \rightarrow \infty} \operatorname{Pr}\left\{\sigma^{t} \in S\right\}=1$. Proof. According to Young (1998) in any potential game with the log-linear behavioural rule, the set of all the states that maximise the potential function is stochastically stable. As in our model $\Phi$ is the potential function, so the states that maximise $\Phi$ are stochastically stable. 
Proposition 2. In the long run, if $\beta$ is large and $\alpha$ is sufficiently large then residential integration is observed almost all the time.

Proof. By proposition 1 we know that when $\beta$ is large in the long run we will observe almost all the time a state that maximises $\Phi$. The characteristics of these states will depend on the actual number of type 1 agents living in a given neighbour: $x_{1}$.

As long as $x_{1}<\tau$ the system will move towards a segregated pattern because consumption is not affected, but as long as segregation increases the probability of reaching a situation where $x_{1} \geq \tau$ will increase too. When $x_{1} \geq \tau$, there are two forces going in opposite directions. On one hand, the location utility will decrease with the number of different type agents neighbouring each other. On the other hand the consumption utility will decrease with the number of type 1 agents living together in the same neighbourhood. The first term of expressions 10,11 and 14 corresponds to this first effect meanwhile the second term of them corresponds to the second one. The first terms of expressions 10,11 and 14 are clearly positive. As $(n+b) \leq 2 n,(n+a) \leq 2 n$ and $\tau<2 n$ and as the first part of the second terms of expressions 10 and 11 is less than $C$, the whole of the second terms of expressions 10 and 11 are negative. In expression 14 as $c>d$ the first term of the equation is positive and the second one is negative because $\tau<2 n$.

Hence, which effect will predominate depends on the value of $\alpha$. When $\alpha$ is sufficiently large then the second term of those equations will be larger than the first one and consequently the sign of the complete expressions will be negative, which means that the consumption utility will be so important that its reduction will hurt agents and hence they will not be interested in swapping their locations. Under these conditions the states that maximises $\Phi$ are those that minimise segregation.

\section{Simulations}

The aim of our simulations is twofold: firstly, to analyse $\alpha$ and its relationship with a nonsegregated pattern. Specifically we want to observe the predictions made by Proposition 2 that the system will converge towards non-segregated equilibria as long $\alpha$ increases to big enough values. Secondly, to compare the aggregated utility reached in equilibrium with the highest level of aggregated utility, as a way to look at the social optimality of segregation.

\subsection{Equilibrium segregation}

We have formally shown that integration can be a stable equilibrium. Now we will examine some features of an equilibrium of these characteristics using simulations. As it was mentioned, Singh et al. (2009) indicate that segregation is a small city phenomenon, consequently, as we are looking for integration equilibria (as our formal results establishes it will arise when consumption is of high relative importance) we run simulations using a small city because in this situation is more probable to observe segregation equilibria, which is an adverse scenario for the equilibria we are looking for. For simulation purposes, an artificial society comprising 100 agents is considered. Hence, $N=10$. Both types of agent are equally distributed across the population, therefore $\pi_{0}=\pi_{1}=0.5$. Moore local neighbourhoods 
were considered for every agent, which include the eight adjacent agents as neighbours, with a total amount of 9 agents per neighbourhood. Zhang (2004) shows, that models using this kind of neighbourhood converge faster, and moreover the final outcome is independent of the neighbourhood type ${ }^{2}$. Two scenarios will be analysed. In the first one it is assumed that non-disadvantaged agents and disadvantaged ones prefer balanced neighbourhoods. Consequently, $n_{1}=n_{0}=4$. In the second one we study the system behaviour when nondisadvantaged agents have strong preferences for living among peers, then $n_{0}=8$. In order to facilitate the calculations and normalize the utility functions, we set $Z=1$ and $M=0.6$. Chosen values for parameters are $\beta=10$ and $\tau=5$, consequently the function simulated is $10(\ell+\alpha C)+\epsilon$. $\beta$ determines the extent of importance of the non-stochastic part of the utility function. $\beta$ equal to zero implies all agents moving in a random way. The Appendix shows a pseudo-code of the algorithm.

We started the simulations with $\alpha=0$ and then we incremented $\alpha$ by 0.02 until we reached $\alpha=0.7$ (therefore $10 \alpha=7$ ). For each value of $\alpha$ we performed 10 runs of 3,000 simulations each of them, and then we calculated the average segregation. Figure 6 depicts the relationship between $\alpha$ and the average segregation when $n_{0}=4$ and Figure 7 shows the same but when $n_{0}=8$. Regarding $\alpha$ our focus is on how important is consumption relative to location, therefore we are not interested in the value of $10 \alpha$ itself but in how many times is consumption greater than location preferences.

As it can be appreciated, in the first case, for values between 0 and 4, the system converges to high levels of segregation (the segregation measure has been normalised between 0 and 1). Once $10 \alpha$ exceeds this threshold, segregation falls pronouncedly. When $10 \alpha=5.7$, the system always converges to the lowest possible level of segregation: 0.04 . The latter is the level of segregation that the system produces with the first random move at the startup of simulations.

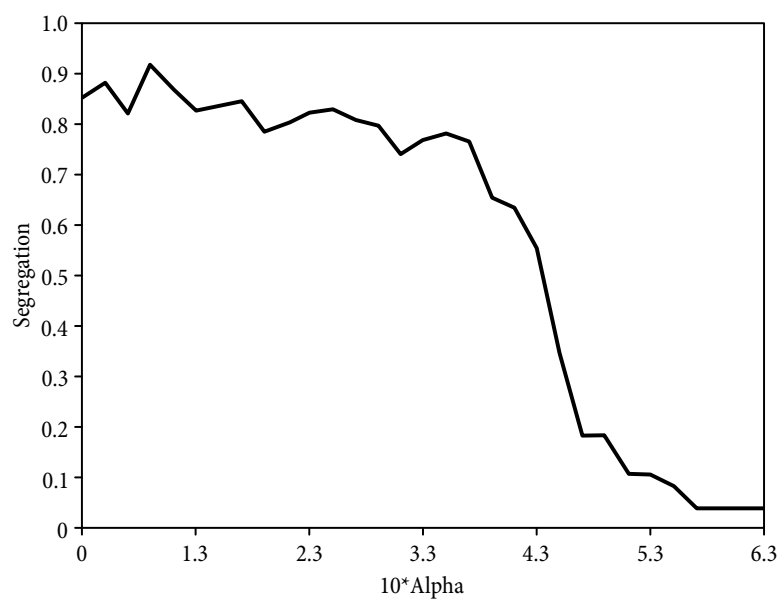

Fig. 6. Segregation equilibria with $n_{0}=4$

\footnotetext{
2 The other two kind of neighbourhoods used in the agent-based literature are Von Neumann and $r(2)$, where the former considers the four surrounding agents as neighbours and the latter comprises 12 agents inside a circle with radius 2 .
} 


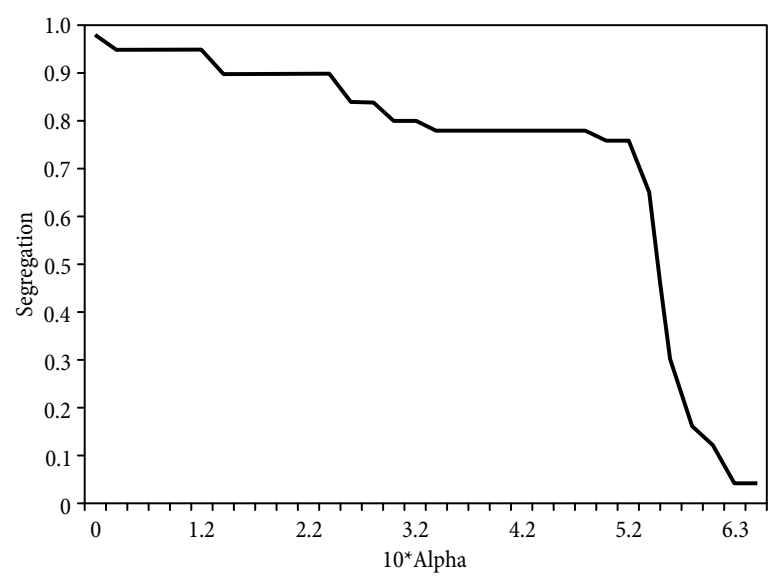

Fig. 7. Segregation equilibria with $n_{0}=8$

The system behaviour in the second case is very similar (Fig. 7), but the high level of segregation can be observed for greater values of $\alpha$. As a matter of fact for values of $10 \alpha$ between 0 and 5.5 the average segregation is greater than 0.7 . Once $10 \alpha$ goes beyond 5.5 the average segregation falls, and after the value of 6.3 has been reached, the system stabilises at an average segregation of 0.04 .

The fact that the system stabilises at 0.04 means that full integration comes to be a stable equilibrium. This finding is quite different from the previous results reported in literature, where the main conclusion has been exactly the opposite: the system always converges to the highest level of segregation. The element that makes the difference is that here, agents take into account segregation's negative effect on their own level of consumption. This result will remain as long as consumption is important enough compared to location characteristics as Proposition 2 indicates.

\subsection{Well-being analysis}

In this section we compare equilibrium segregation with the segregation that produces the highest aggregated utility for a given set of parameter values. We call the segregation related to the maximum level of aggregated utility Optimal Segregation (in the Appendix there is an explanation of the algorithm used for this propose). The analysis has been made for a different combination of parameters values. In particular the focus is on non-disadvantaged agents' prejudice $n$ and on the effects of segregation on productivity of disadvantaged agents $\tau$. Values considered for $n$ are 5,6,7 and 8, and for $\tau$ we considered 4, 5, 6, 7 and 8 , i.e. we combine every level of prejudice, $n$, with all possible levels of segregation's negative impact on productivity $\tau$, and for every one of these combinations we have obtained optimal segregation and equilibrium segregation, and then we calculated the difference between these two values. We made this exercise for two situations, one, when $\alpha$ is closer to 0 , consequently the system evolves to full segregated equilibria and, second, when $\alpha$ is greater than 0.65 (consequently $10 \alpha$ is greater than 6.5 ), which makes the system evolve to 
non-segregated equilibria. Figures 8 and 9 present the average difference between Equilibrium Segregation (ES) and Optimal Segregation $(O S)$ for all analysed cases.

As mentioned, when $\alpha$ is closer to 0 the system evolves to full segregated equilibria, however as $\alpha$ is greater than 0 segregation produces a loss in utility due to the consumption decrease. Hence, to find out when ES becomes optimal, we must ask when full segregation is optimal. The answer is linked to the non-disadvantaged agents' level of prejudice and the extent of the segregation impact on the productivity of the disadvantaged agents. Then, the higher the prejudice, the higher the level of optimal segregation, and the lower the impact on productivity, the lower the level of optimal segregation. In this case there is only one situation where equilibrium and optimal segregation coincide, that is, when the latter is also the highest level of segregation. Without any surprise, this result comes about when non-disadvantaged agents are highly prejudiced and the negative impact of segregation upon the disadvantaged agents' productivity is irrelevant. In Figure 8 it is possible to appreciate that when $n_{0}=8$, i.e. when advantaged agents want to live just with like neighbours - maximum prejudice - and $\tau=8$, i.e. when there is no impact of segregation on productivity, equilibrium segregation and optimal segregation are equal, hence $E S-O S=0$. The opposite case occurs when prejudice reaches a value of 5 and the impact of segregation on productivity reaches its maximum $(\tau=4)$. In that case, the difference between equilibrium segregation and optimal segregation reaches its maximum for any level of prejudice.

These results indicate that in almost all cases, society is in a situation where the optimal level of aggregate utility is not reached. A problem that increases meanwhile the level of non-disadvantaged agents' prejudice decreases. This is because when the level of prejudice decreases, the difference between the optimal segregation and the equilibrium segregation gets higher. The intuition is straightforward: less prejudiced agents prefer lower levels of segregation, then the optimal level of segregation will be lower too, but the equilibrium segregation remains at the same high level, hence the social loss, under these circumstances, is bigger.

When $\alpha$ is big enough the system evolves to low level of segregation equilibria. In this case, as it can be seen in Figure 9, difference between ES and OS are very small, particularly when $\tau$ is 4 and 5, the highest impact of segregation on productivity. Gradually the gap gets bigger, till $\tau=8$ when the difference reaches the maximum value. The reason is that when $\alpha$ is greater than 0.65 the system stabilises at very low levels of segregation, but if $\tau=8$ segregation almost does not affect productivity, therefore some positive values of segregation will increase the aggregated utility.

The extreme cases where either the highest or the lowest segregation are the social optimum, sort of corner solutions, are less common. This implies that segregation reduction can generate social gains, but, in almost all cases, not its complete elimination. In other words, segregation is not bad by itself, but it is not good either just because it is an equilibrium state. To search for policies which can establish a socially optimal individual allocation, demands a full understanding of agents' preferences for local neighbourhoods characteristics and the extent of groups effect impact upon disadvantaged agents. Therefore neither a full elimination of segregation nor a laissez-faire equilibrium can be seen a priori as the best solution. 


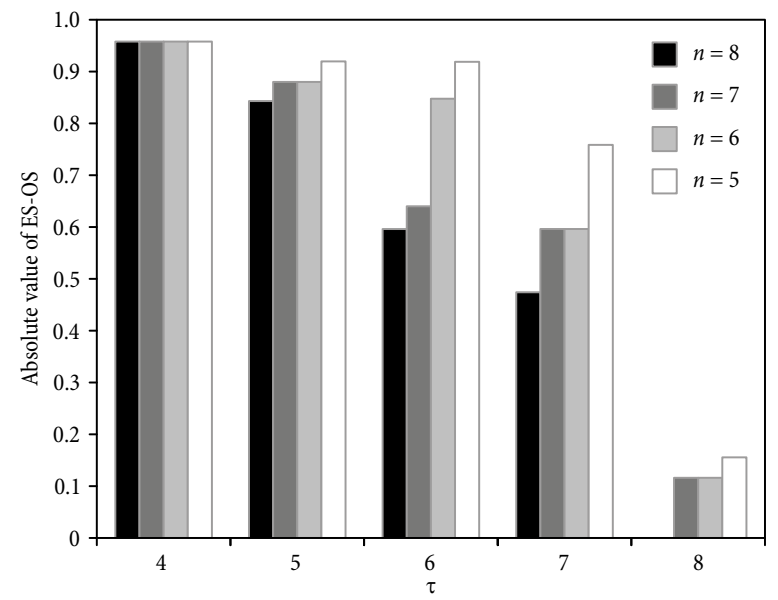

Fig. 8. Difference between $E S$ and $O S$ for $\alpha$ close to 0

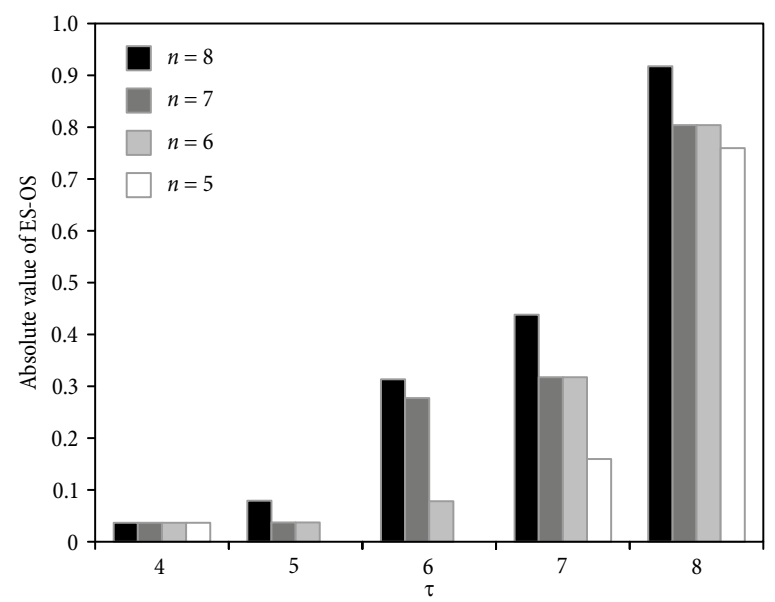

Fig. 9. Difference between $E S$ and $O S$ for $10 \alpha>6.5$

\section{Conclusions}

The well known Schelling model predicts that segregation is the unique stable equilibrium even when individuals have preference for living in integrated communities, which has been confirmed by subsequent investigations. Nevertheless, it is empirically possible to observe that segregation varies across cities and even in highly segregated cities there is some extent of intermingle. We argue that this difference between theoretical prediction and reality emerges because individuals do not take into account just their neighbours characteristics when they decide where to live but other factors as well, such as, for instance, public goods consumption.

Based on this concept, in this paper we have developed a mathematical formalisation of a Schelling-like model of segregation, which includes a simple production function. The main difference from the previous literature is that here we consider that segregation can 
affect the utility of every single agent, including non-segregated ones. There are two types of agents, advantaged and disadvantaged. The novelty of this work arises in the assumption that disadvantaged agents living segregated lose productivity and then the economy as a whole is less productive too, affecting in a negative way the level of consumption of all agents.

Segregation also affects production negatively and therefore the levels of consumption of everyone, prejudiced and non-prejudiced alike. The mechanism driving this process is that of group effects.

Using this model and stochastic evolutionary game theory techniques we prove that when individuals' consumption is important enough, integration can emerge as a stable equilibrium. The reason is that agents take into account segregation's negative effects on their own level of consumption at the moment of taking their location decisions. This is an interesting result because is the first time that has been formally shown that integration can be a stable equilibrium.

After a formal proof was obtained, two simulation scenarios were analysed: one where both kinds of agents prefer balanced neighbourhoods, and a second one where advantaged agents have strong preferences for neighbouring just peers. Under these two scenarios we observed that when $\alpha$ is big enough the system converges to a full integration equilibrium. The only difference is that in the second case the value of $\alpha$ needed for observing integration is greater than the one needed in the first case.

This result can be interpreted as follows: when individuals are aware about the potentially pernicious effects of segregation, which can affect every single society member, they are more conscious at the moment of choosing their location. Depending on how detrimental is segregation, or how important the location characteristics are, the equilibrium segregation will vary from high levels to almost full integration, which is something that can be empirically observed: according to The Social Science Analysis Network calculations, based on census data, the average dissimilarity index for White and Black individuals in the U.S.A. is 58.5 (the maximum value is 100).

We also use simulations for comparing the aggregated utility of the optimal segregation level with equilibrium segregation one. The optimal segregation value depends on the degree of prejudice and on its negative impact of group effects on productivity. For instance, with highly prejudiced agents and irrelevant group effects, the social optimum will be full segregation. In the opposite case, the social optimum will be full integration. However, with more balanced parameter values, the optimal segregation will be a positive value lying between 0 and 1 but not reaching the extremes.

Although we are aware that more research is needed before proposing any kind of policy implications, our findings can suggest something. First of all, segregation must not be seen as a bad situation a priori. As a matter of fact, using the model presented in this work, it has been possible to find different levels of optimal segregation, from no segregation at all up to full segregation. What is going to be the optimal level of segregation is something which depends on how prejudiced are the prejudiced agents, the extent of the impact of group effects, and the relative importance of local neighbourhood characteristics and consumption on agents' utilities. Consequently, it can be argued that there is no such 
thing as a unique optimal level of segregation or an absolute segregation target. In other words, segregation is not bad in itself, because there is one part of society that wants to live segregated, but it is not good in itself either, because segregation affects all agent's levels of consumption. If the aim is to improve welfare, policy makers must have a clear picture of individual preferences, group effects, and other elements, which have not been treated in this research, but it is worth mentioning them, such as the provision of local and non-local public goods, before implementing any policy with the objective of reducing segregation. Besides, it is clear too that if two cities, or regions, have not the same characteristics, then they have different levels of optimal segregation and, therefore, they need different policies.

Despite the fact that the model developed in the present paper has provided interesting insights into segregation, it is also true that is a simple model. So that further work may be needed to obtain a deeper understanding of this phenomenon. For instance, incorporating prices, the real estate market, the labour market and the capital market. Using a model like that, the relationship between segregation - group effects - and economic growth could be investigated. It is worthwhile also to empirically investigate on the elements defining the level of optimal segregation, such as prejudices and group effects, as a way to test the findings of the present paper, and, also, as a useful base for policy design.

\section{References}

Alden, L.; Hammarstedt, M.; Neuman, E. 2014. Ethnic segregation, tipping behavior, and native residential mobility, International Migration Review 49(1): 36-69. http://dx.doi.org/10.1111/imre.12066

Ananat, E. 2011. The wrong side(s) of the tracks: the caussal effects of racial segregation on urban poverty, American Economic Journal: Applied Economics 3(2): 34-66.

Bayer, P.; McMillan, R.; Rueben, K. 2004. What drives racial segregation, Journal of Urban Economics 56: 514-535. http://dx.doi.org/10.1016/j.jue.2004.06.002

Bayer, P.; Fang, H.; Mcmillan, R. 2014. Separate when equal? Racial inequality and residential segregation, Journal of Urban Economics 82: 32-48. http://dx.doi.org/10.1016/j.jue.2014.05.002

Benabou, R. 1996. Heterogeneity, stratification, and growth: macroeconomic implication of community structure and school finance, American Economics Review 86(3): 584-609.

Bischi, G.; Merlone, U. 2011. An adaptative dynamic model of segregation, in T. Puu, A. Panchuk (Eds.). Nonlinear Economic Dynamic. Nova Science Publishers, 191-205.

Bjerk, D. 2010. Thieves, thugs, and neighborhood poverty, Journal of Urban Economics 68(3): 231-246. http://dx.doi.org/10.1016/j.jue.2010.06.002

Bolt, G.; Ozuekren, S.; Phillips, D. 2010. Linking integration and residential segregation, Journal of Ethnic and Migration Studies 36(2):169-186. http://dx.doi.org/10.1080/13691830903387238

Clampet-Lundquist, S.; Massey, D. 2008. Moving to opportunity: a symposium Neighborhood Effect on Economic Self-Sufficiency: A Reconsideration of the Moving to Opportunity Experiment, American Journal of Sociology 114(1): 107-143. http://dx.doi.org/10.1086/588740

Clark, W. A. 1991. Residential preferences and neighborhood racial segregation: a test of the Schelling segregation model, Demography 28(1): 1-19. http://dx.doi.org/10.2307/2061333

Conejeros, R.; Vargas, M. 2007. Group effect, productivity and segregation optimality, Lecture Notes in Economics and Mathematical Systems 599: 209-222. http://dx.doi.org/10.1007/978-3-540-73135-1_15

Corvalan, A.; Vargas, M. 2015. Segregation and conflict: an empirical analysis, Journal of Development Economics 116: 212-222. http://dx.doi.org/10.1016/j.jdeveco.2015.05.002 
Grauwin, S.; Bertin, E.; Lemoy, R.; Jensen, P. 2009. Competition between collective and individual dynamics, Proceedings of the National Academy of Sciencies of United States of America 106(49): 20622-20626. http://dx.doi.org/10.1073/pnas.0906263106

Grauwin, S.; Goffette-Nagot, F.; Jensen, P. 2012. Dynamic models of residential segregation: an analytical solution, Journal of Public Economics 96: 124-141. http://dx.doi.org/10.1016/j.jpubeco.2011.08.011

Kling, J.; Liebman, J. 2004. Experimental analysis of neighborhood effects on youth. Working Paper, Princeton IRS.

Kling, J.; Liebman, J.; Katz, L. 2007. Experimental analysis of neighborhood effects, Econometrica 75(1): 83-119. http://dx.doi.org/10.1111/j.1468-0262.2007.00733.x

Kling, J.; Ludwig, J.; Katz, L. 2005. Neighborhood effects on crime for female and male youth: evidence from a randomized voucher experiment, Quarterly Journal of Economics 120(1): 87-130.

Ludwig, J.; Duncan, G.; Gennetian, L.; Katz, L.; Kessler, R.; Kling, J.; Sanbonmatsu, L. 2013. Long-term neighborhood effects on low-income families: evidence from moving to opportunity, American Economic Review: Papers and Proceedings 103(3): 226-231. http://dx.doi.org/10.1257/aer.103.3.226

Ludwig, J.; Kling, J.; Sanbonmatsu, L.; Liebman, J.; Duncan, G.; Kessler, R. 2008. What can we learn about neighborhood effects from the moving to opportunity experiment?, American Journal of Sociology 114(1): 144-188. http://dx.doi.org/10.1086/588741

McFadden, D. 1973. Conditional logit analysis of qualitative choice behavior, in P. Zarembka (Ed.). Frontiers of econometrics. New York: Academic Press, 105-142.

Monderer, D.; Shapley, L. 1996. Potential games, Games and Economic Behavior 14(44): 124-143. http://dx.doi.org/10.1006/game.1996.0044

Pancs, R.; Vriend, N. 2007. Schelling's spatial proximity model of segregation revisited, Journal of Public Economics 91: 1-24. http://dx.doi.org/10.1016/j.jpubeco.2006.03.008

Ruoff, G. 2006. Segregation in the classroom: an empirical test of the schelling model, Rationality and Society 18(1): 95-117. http://dx.doi.org/10.1177/1043463106060154

Schelling, T. 1971. Dynamics models of segregation, Journal of Mathematical Sociology 1: 143-186. http://dx.doi.org/10.1080/0022250X.1971.9989794

Singh, A.; Vainchtein, D.; Weiss, H. 2009. Schelling's segregation model: parameters, scaling, and aggregation, Demographic Research 21: 341-366. http://dx.doi.org/10.4054/DemRes.2009.21.12

Xie, Y.; Zhou, X. 2014. Modeling individual-level heterogeneity in racial residential segregation, Proceedings of the National Academy of Sciencies of United States of America 109(29): 11646-11651. http://dx.doi.org/10.1073/pnas.1202218109

Young, H. P. 1998. Individual strategy and social structure: an evolutionary theory of institutions. Princeton, New Jersey, USA: Princenton University Press. 208 p.

Zhang, J. 2004. Residential segregation in an all-integrationist world, Journal of Economic Behavior and Organization 54(4): 533-550. http://dx.doi.org/10.1016/j.jebo.2003.03.005

Zhang, J. 2011. Tipping and residential segregation: a unified schelling model, Journal of Regional Sciences 51(1): 167-193. http://dx.doi.org/10.1111/j.1467-9787.2010.00671.x

Zhang, J.; Zheng, L. 2015. Are people willing to pay for less segregation? Evidence from U.S. internal migration, Regional Science and Urban Economics 53: 97-112.

http://dx.doi.org/10.1016/j.regsciurbeco.2015.05.002 


\section{APPENDIX}

\section{Pseudo-code}

\begin{tabular}{|c|c|}
\hline \multicolumn{2}{|r|}{ Algorithm 1 Household exchange algorithm procedure } \\
\hline 1: & procedure HOUSEHOLD EXCHANGE \\
\hline 2: & Set initial neighbourhood distribution as full integration \\
\hline 3: & Perform initial random house swap \\
\hline 4: & while maximum iteration criterion not satisfied \\
\hline 5: & * Select at random two households for exchange \\
\hline 6: & ${ }^{*}$ Compute utility $(U)$ for each household and location \\
\hline 7: & * Add random externalities to $U(U=U+\varepsilon)$, in order to account \\
\hline & for mistakes and heterogeneity in decision making \\
\hline 8: & $\begin{array}{l}\text { if } \sum_{i=1,2} U_{i} \text { at location } 2>\sum_{i=1,2} U_{i} \text { at location } 1 \text {, where } i \text { indicates } \\
\text { households' location then }\end{array}$ \\
\hline 9: & * Perform exchange \\
\hline 10: & end if \\
\hline 11: & Report results \\
\hline 12: & end while \\
\hline 13: & end procedure \\
\hline
\end{tabular}




\begin{tabular}{|c|c|}
\hline \multicolumn{2}{|r|}{ Algorithm 2 Optimal Segregation Algorithm } \\
\hline 1: & procedure OPTIMAL SEGREGATION \\
\hline 2: & Set random initial neighbourhood distribution \\
\hline 3: & Set Social Utility $(S U)$ \\
\hline 4: & Set Total Productivity $(T P)$ to 0 \\
\hline 5: & Let $S U_{\text {best }_{\text {out }}}=S U_{\text {best }_{\text {in }}}=S U$ \\
\hline 6: & $T P_{\text {best }_{\text {out }}}=T P_{\text {best }_{\text {in }}}=T P$ \\
\hline 7: & while maximum outer iteration criterion not satisfied do \\
\hline 8: & while maximum inner iteration criterion not satisfied do \\
\hline 9: & Select at random two households for exchange \\
\hline 10: & Compute utility $(U)$ for each household and location \\
\hline 11: & $\begin{array}{l}\text { Add random externality to } U(U=U+\varepsilon \text {, in order to account } \\
\text { for mistakes and heterogeneity in decision making }\end{array}$ \\
\hline 12: & If $U$ at location $2>U$ at location 1 then \\
\hline 13: & Perform household exchange \\
\hline 14: & Compute $S U$ and $T P$ \\
\hline 15: & end if \\
\hline 16: & end while \\
\hline 17: & if $S U_{\text {best }_{\text {in }}}<S U$ then \\
\hline 18: & $S U_{\text {best }_{\text {in }}}=S U$ \\
\hline 19: & $T P_{\text {best }_{\text {in }}}=T P$ \\
\hline 20: & end if \\
\hline 21: & if $S U_{\text {best }_{\text {out }}}<S U_{\text {best }_{\text {in }}}$ then \\
\hline 22: & $S U_{\text {best }_{\text {out }}}=S U_{\text {best }_{\text {in }}}$ \\
\hline 23: & $T P_{\text {best }_{\text {out }}}=T P_{\text {best }_{\text {in }}}$ \\
\hline 24: & end if \\
\hline 25: & end while \\
\hline 26: & Report results $S U_{\text {best }_{\text {out }}}$ and $T P_{\text {best }_{\text {out }}}$ \\
\hline 27: & end procedure \\
\hline
\end{tabular}


Raúl CONEJEROS, Full Professor at Escuela de Ingeniería Bioquímica, Pontifical Catholic University of Valparaíso, Chile. PhD in Chemical Engineering, MSc in Biochemical Engineering UCV. Author and co-author of more than 20 scientific publications and 2 book chapters. Research interests are in the application of non-linear optimization and optimal control techniques to biochemical engineering systems and to life cycle analysis. Collaboration with Dr. Vargas has led to research interests in economy and urban planning.

Miguel VARGAS, Associated Professor at Department of Economics, Universidad Diego Portales, Chile. PhD and Msc in Economics. He is author of several articles and book chapters. Research interests are in Urban Economics, Social Dynamics and Industrial Organisation. He has been visiting scholar at Department of Land Economy, University of Cambridge and consultant to Inter American Bank of Development and The Lincoln Institute of Land Policy. 